\title{
Role of Fluid Management on Renal Failure in Hospitalized COVID-19 Patients
}

\author{
Angelie Santos, Hillary Grainer, Joseph Scarano, Majed Samarneh \\ Department of Internal Medicine, St. John's Riverside Hospital, New York, USA \\ Email: anniesantos0815@gmail.com
}

How to cite this paper: Santos, A., Grainer, H., Scarano, J. and Samarneh, M. (2021) Role of Fluid Management on Renal Failure in Hospitalized COVID-19 Patients. Open Journal of Nephrology, 11, 230-241. https://doi.org/10.4236/ojneph.2021.112018

Received: April 14, 2021

Accepted: May 23, 2021

Published: May 26, 2021

Copyright (c) 2021 by author(s) and Scientific Research Publishing Inc. This work is licensed under the Creative Commons Attribution International License (CC BY 4.0).

http://creativecommons.org/licenses/by/4.0/

(c) (i) Open Access

\begin{abstract}
Introduction: The reported incidence of AKI with COVID-19 varies from $0.5 \%$ to $22 \%$. Several mechanisms were postulated as a cause of AKI in patients infected with COVID-19. The appropriate management of AKI in patients with COVID-19 remains unclear at this time. One point of absolute importance, is the consideration of volume status. Given the paucity of knowledge with regards to the role of different strategies for fluid management during an episode of AKI in patients with COVID-19, this retrospective study aims to compare renal outcome and overall prognosis in patients who received conservative versus liberal fluid management. Methods: This is a single-center retrospective observational cohort study at a community hospital in Westchester County, NY. All adult patients who tested positive for the COVID-19 infection by PCR testing of a nasopharyngeal swab and were hospitalized from March 22, 2020 to May 25, 2020 are eligible. Among those identified with AKI, patients were divided into two groups: conservative fluid administration versus liberal fluid administration. Results: Of the 136 patients, $84(61.76 \%)$ were admitted to the ICU, with $60 \%$ of patients under the conservative fluid strategy and $40 \%$ receiving liberal fluid management. On the other hand, 52 (38.23\%) patients were admitted on the medical floors, with more patients $(67.31 \%)$ receiving liberal fluid management. Discussion: In our cohort of 136 patients with COVID-19 respiratory illness and AKI, there was a significant difference in renal outcome, in terms of improvement of renal function in patients receiving liberal fluid management $(55.07 \%)$ versus conservative fluid management $(16.41 \%, \mathrm{p} \leq 0.001)$, with more patients in the liberal group having lower peak creatinine before levels improved. This, as well, was associated with improvement in oxygenation, characterized by improvement in respiratory status, facilitating weaning of oxygen supplementation $(\mathrm{p}<0.001)$. On the other hand, there was no significant difference between the conservative and liberal groups in terms of undergoing renal replacement therapy. Twenty-one of the 136 patients with AKI
\end{abstract}


required RRT and 19 (90\%) of them were admitted to the ICU and mechanically ventilated. On the other hand, there was no statistical difference in mortality rate of patients who underwent renal replacement therapy, regardless of whether they were in the conservative or liberal strategy group. Conclusion: Our data report that liberal fluid management in COVID-19 patients with AKI, had better outcomes, in terms of renal function, oxygenation and mortality rate, as compared to patients in the conservative fluid management group. Once patients are started on renal replacement therapy, however, renal and lung outcomes and mortality rate become insignificant between the two groups.

\section{Keywords}

Acute Kidney Injury, COVID-19, Fluid Management

\section{Introduction}

In December 2019, a series of unknown acute respiratory illnesses were reported in Wuhan, China [1] [2]. Research has shown that the disease was caused by a virus named "severe acute respiratory syndrome coronavirus 2" (SARS-CoV-2) and later on the disease was officially renamed by the WHO as coronavirus disease 2019 (COVID-19). Since it was first recognized, COVID-19 has rapidly spread throughout other countries and became a worldwide pandemic [3] [4] [5]. In late January 2020, the United States reported its first case of COVID-19 in Washington State. And shortly after, spread to other states, including New York where it became the epicenter of the coronavirus outbreak in the U.S., reporting the most number of cases with 386,490 and 30,511 reported deaths as of June 12, 2020. As had been observed in China and Italy, the disease resulted in a large number of hospitalizations, respiratory failure and ICU admissions. Although diffuse alveolar damage and acute respiratory failure were the main features of COVID-19, multi organ involvement have been reported. The kidneys, in particular became noticeably affected, with an alarming number of patients developing AKI [6] [7].

The reported incidence of AKI with COVID-19 varies from $0.5 \%$ to $22 \%$ [6] [7] [8]. This wide range is likely in part to the definition of AKI used and to the population studied. U.S. data in a Seattle hospital reported up to $19 \%$ rate of AKI, though this has been limited to critically ill patients in the ICU [9]. In a study released by Northwell Health, it showed $36.6 \%$ of patients admitted with COVID-19 developed AKI, with $89.7 \%$ of patients on mechanical ventilation compared to $21.7 \%$ of non-ventilated patients. Ninety-six percent of patients requiring RRT on ventilators. It was also noted in this study that AKI occurs early and in temporal association with respiratory failure and is associated with a poor prognosis [10] [11]. A single-center retrospective study by Zahid et al. revealed that $27.3 \%$ of patients hospitalized for COVID-19 developed AKI, with 
the incidence significantly higher in patients presenting with a low eGFR, and carried higher mortality, especially in patients with AKI stage 3 [12].

Several mechanisms were postulated as a cause of AKI in patients infected with COVID-19. It is believed to be an interplay of virus-mediated injury, a dysregulated inflammatory response, angiotensin II pathway activation, hypercoagulation, and microangiopathy [12]. The appropriate management of AKI in patients with COVID-19 remains unclear at this time. No specific evidence is available to suggest that COVID-19 AKI should be managed differently from other causes of AKI [13]. No single approach has demonstrated clear benefit for patients who have AKI and develop respiratory failure. One point of absolute importance is the consideration of volume status. It is known from existing knowledge about ARDS management, that a conservative approach to fluid resuscitation and efforts to avoid hypervolemia would be appropriate. A post hoc analysis of the FACTT trial supports the use of conservative strategy of fluid management in patients with acute lung injury, as it demonstrated that a negative fluid balance using a higher cumulative dose of diuretics is associated with improved mortality in patients with AKI [14]. In this regard, diuresis may have been a key component in the treatment of patients with Covid-19. Overdiuresis, however, has the potential to exacerbate kidney injury, with several features of this disease magnifying the risk including significant volume contraction from poor oral intake and insensible fluid losses, and prolonged ventilator dependence resulting in prolonged diuresis [15]. As time passed, and more patients were hospitalized, it was noted that severe AKI became more prominent, with increasing number of patients requiring renal replacement therapy. Since then, attempts have been made to prevent worsening AKI by providing liberal intravenous fluids where fluids are indicated.

Given the paucity of knowledge with regards to the role of different strategies for fluid management during episode of AKI in patients with COVID-19, this retrospective study aims to compare renal outcome and overall prognosis in patients who received conservative versus liberal fluid management. In addition, identify if there was a significant change in patients' oxygenation with conservative or liberal fluid management.

\section{Methods}

\subsection{Study Design}

This is a single-center retrospective observational cohort study at a community hospital in the Westchester county, the second most affected area after New York City. Data for this study was obtained from Meditech, the inpatient electronic medical record of St. John's Riverside Hospital. This study was approved by the Institutional Review Board of St. John's Riverside Hospital and did not require patient consent because of its retrospective design.

\subsection{Inclusion Criteria}

All adult patients ( $\geq 18$ years old) who tested positive for the COVID-19 infec- 
tion by PCR testing of a nasopharyngeal swab and were hospitalized from March 22, 2020 to May 25, 2020 are eligible. COVID-19 infection was diagnosed based on clinical presentation, radiographic lung abnormalities, and a positive result of real time PCR. Eligible patients included those who developed Acute Kidney Injury, as defined by the KDIGO criteria, with previously normal or baseline creatinine dating back to one year prior to hospitalization. AKI secondary to insensible losses such as dehydration, GI disturbances, and mechanical ventilation were included.

\subsection{Exclusion Criteria}

Patients with End Stage Kidney Disease on maintenance hemodialysis or peritoneal dialysis were excluded from the study. Also excluded were patients who died within 24 hours of admission and renal transplant recipients.

\subsection{Study Procedure}

We extracted electronic data from all patients who satisfy the inclusion criteria, admitted both in the ICU and medical floors. Data collected include patient demographics, prior medical history, including comorbidities and baseline renal function. In-hospital laboratory data consisted of renal function, initial $\mathrm{Na}$ level, ABGs. For patients in the ICU, pressor administration and mechanical ventilation were identified. Total fluid volume (in liters) administered from the emergency room, on admission, and during the hospital course were noted. Among those identified with AKI, patients were divided into two groups: conservative fluid administration verses liberal fluid administration.

Acute kidney injury is defined as an increase in serum creatinine $0.3 \mathrm{mg} / \mathrm{dl}$ within 48 hours or a $50 \%$ increase in serum creatinine from baseline within 7 days according to the KDIGO criteria. The date of AKI onset is defined as the earliest day of a serum creatinine change meeting KDIGO criteria. The stage of AKI is determined using the peak serum creatinine level after AKI detection, with increases of 1.5 to $1.9,2.0$ to 2.9 , and $\geq 3$ times baseline being defined as AKI stage 1,2 , and 3 , respectively.

Primary outcome was the improvement/worsening of renal function with conservative/liberal fluid management. Conservative fluid management being defined as no maintenance fluids and administration of diuretics where it is indicated and liberal fluid management being fluid boluses and maintenance fluids of $2 \mathrm{cc} / \mathrm{kg} / \mathrm{hr}$.

\section{Objective}

The primary objective of this study was to evaluate the role of fluid therapy on renal outcome in patients infected with COVID-19 associated with AKI, to compare renal outcome and overall prognosis in patients who received conservative versus liberal fluid management. The secondary objective of the study was to evaluate the outcome of fluid therapy on oxygenation through ABG analysis. In addition, to study baseline characteristics and laboratory data associated with 
the development of AKI in COVID-19 patients.

\section{Statistical Analysis}

Continuous variables were described as mean \pm standard deviation for normally distributed data, or median and interquartile range (IQR) values for non-normal distribution. Categorical variables were presented as frequency and percentage. The mean of continuous variables was assessed by using independent $t$ tests and the Kruskal-Wallis test (across the stages of AKI). The differences in categorical variables were compared by using the chi-square test. Fischer's exact test was used when there were only limited data available. Two-sided P values of 0.05 or less were considered to indicate statistical significance.

\section{Results}

\subsection{Sample Size and Baseline Characteristics}

In this study, 144 adult confirmed cases of COVID-19 with abnormal renal function were initially reviewed. Of the 144 patients, 5 patients with end stage kidney disease who were on maintenance dialysis and 2 patients who died within 24 hours of admission were excluded from the study. No renal transplant recipients were encountered (Figure 1). One hundred thirty-six patients were used for the analysis, with their baseline characteristics shown in Table 1 . Of the 136 patients, $84(61.76 \%)$ were admitted in the ICU, with $60 \%$ of patients under the conservative fluid strategy and $40 \%$ receiving liberal fluid management. On the other hand, 52 (38.23\%) patients were admitted on the medical floors, with more patients (67.31\%) receiving liberal fluid management.

\subsection{Baseline Characteristics}

The two groups were similar with respect to demographic characteristics with

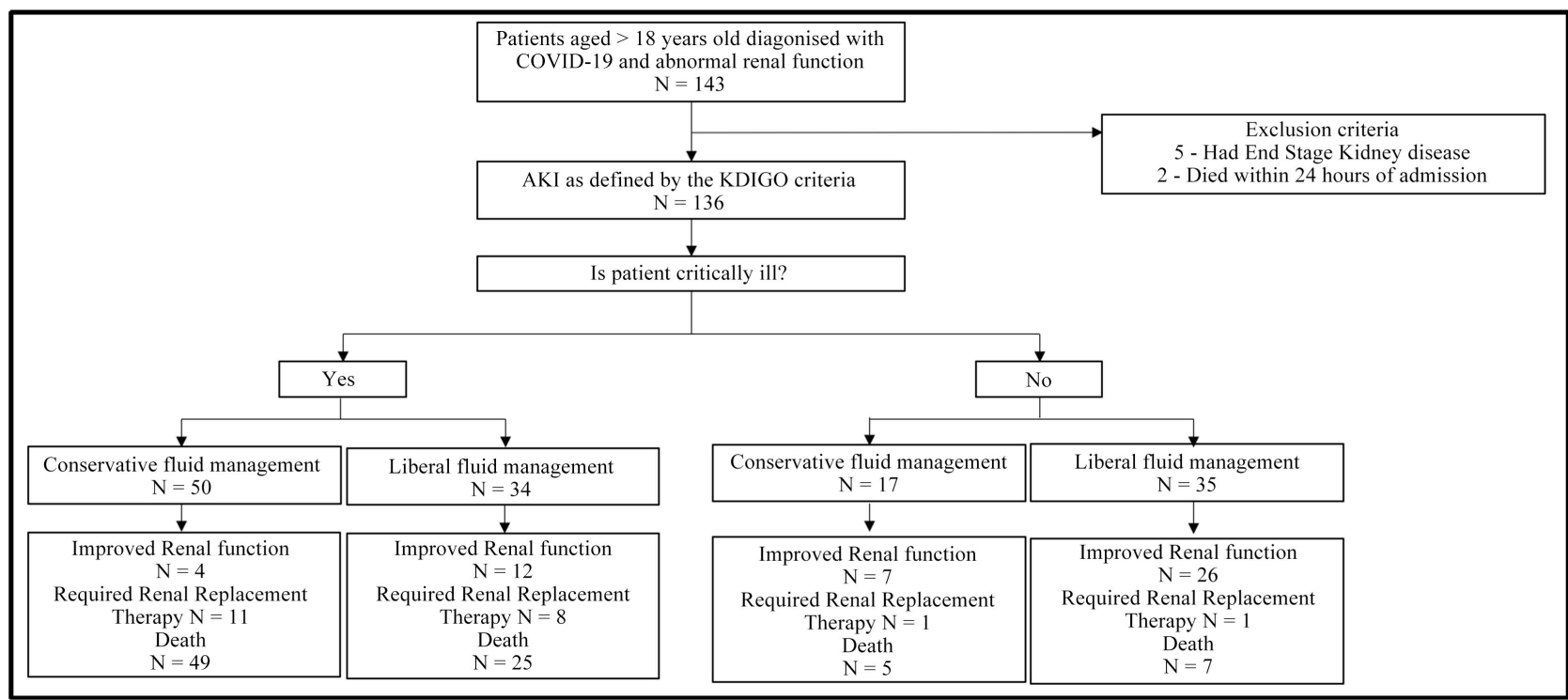

Figure 1. Study flowchat showing the number of participants. 
Table 1. Baseline characteristics.

\begin{tabular}{|c|c|c|c|}
\hline Characteristic & $\begin{array}{l}\text { Conservative strategy } \\
\qquad(\mathrm{N}=67)\end{array}$ & $\begin{array}{l}\text { Liberal strategy } \\
\qquad(\mathrm{N}=69)\end{array}$ & P Value \\
\hline Age, mean (yr) & $72 \pm 11$ & $69 \pm 14$ & 0.29 \\
\hline Age, median (IQR) & $73(66-80)$ & $70(57-83)$ & 0.29 \\
\hline Gender, n (\%) & & & 0.07 \\
\hline Male & $41(61.19)$ & $38(55.07)$ & \\
\hline Female & $26(38.81)$ & $31(44.93)$ & \\
\hline Race, n (\%) & & & 0.48 \\
\hline Hispanic & $25(37.31)$ & $34(49.28)$ & \\
\hline Caucasian & $25(37.31)$ & $19(27.54)$ & \\
\hline African American & $15(22.39)$ & $15(21.74)$ & \\
\hline Asian & $2(2.99)$ & $1(1.45)$ & \\
\hline \multicolumn{4}{|l|}{ Preexisting comorbidities, n (\%) } \\
\hline $\mathrm{CHF}$ & $9(13.43)$ & $11(15.94)$ & 0.18 \\
\hline CAD & $22(32.84)$ & $15(21.74)$ & 0.05 \\
\hline Diabetes & $27(40.30)$ & $32(46.37)$ & 0.11 \\
\hline Hypertension & $54(80.60)$ & $57(82.61)$ & 0.17 \\
\hline CKD & $7(10.45)$ & $10(14.49)$ & 0.16 \\
\hline Immunocompromised & $10(14.93)$ & $9(13.04)$ & 0.19 \\
\hline Medical ICU admission, $\mathrm{n}(\%)$ & $50(74.63)$ & $34(49.28)$ & 0.002 \\
\hline Vasopressor requirement & $48(71.64)$ & $30(43.48)$ & $<0.001$ \\
\hline Mechanical ventilation & $45(67.16)$ & $27(39.13)$ & 0.001 \\
\hline AKI, n (\%) & & & 0.001 \\
\hline Stage 1 & $10(14.93)$ & $28(40.58)$ & \\
\hline Stage 2 & $17(25.37)$ & $18(26.09)$ & \\
\hline Stage 3 & $40(59.70)$ & $23(33.33)$ & \\
\hline Peak creatinine levels, mean \pm SD & & & $<0.001$ \\
\hline Stage 1 & $1.73 \pm 0.31$ & $1.70 \pm 0.50$ & 0.84 \\
\hline Stage 2 & $3.08 \pm 0.12$ & $2.82 \pm 0.53$ & 0.12 \\
\hline Stage 3 & $7.31 \pm 2.70$ & $5.97 \pm 2.53$ & 0.05 \\
\hline \multicolumn{4}{|l|}{ Urinalysis, n (\%) } \\
\hline Proteinuria & $53(79.10)$ & $56(81.16)$ & 0.76 \\
\hline Hematuria & $30(44.78)$ & $22(31.88)$ & 0.12 \\
\hline
\end{tabular}

CHF, Congestive Heart Failure; CAD, Coronary Artery Disease; CKD, Chronic Kidney Disease; ICU, Intensive Care Unit; AKI, Acute Kidney Injury; n, mean average; SD, Standard Deviation.

mean age of 71 years old, and $79(58.09 \%)$ of them were male. A majority of our cohort were Hispanic (43.38\%) and Caucasians (32.35\%). The more common comorbidities, as listed on Table 1, were hypertension (81.61\%) and diabetes 
$(43.38 \%)$. Of the 136 patients, $84(61.76 \%)$ patients were in the ICU, with $59.5 \%$ of patients belonging to the conservative fluid management group, and $40.4 \%$ in the liberal fluid strategy. Among patients in the conservative strategy group admitted in the ICU, more patients required vasopressor support (71.64\%) and are mechanically ventilated $(67.16 \%)$, as compared to those who received liberal fluid management.

\subsection{Renal Function}

The conservative strategy group had higher creatinine values at their peak, with more than half of the patients categorized at stage 3 AKI with $59.70 \%$, as compared to the liberal strategy group with only $33.33 \%$ of the patients having stage 3 AKI at their peak and $40.58 \%$ with only stage 1 AKI at their peak $(\mathrm{p}<0.001)$, as seen in Figure 2. Figure 3 compares the peak creatinine levels of all patients included in the study. At the different stages of AKI, the peak creatinine level of patients in the conservative group are greater than those in the liberal group, and the difference widens as the stage increases.

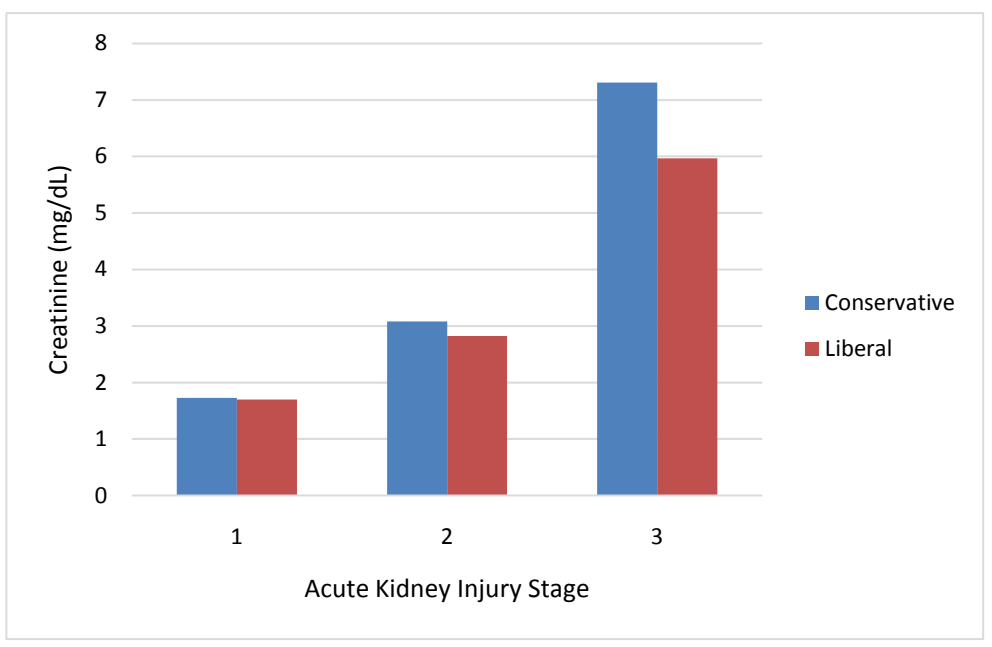

Figure 2. Average Peak Creatinine at different stages of Acute Kidney Injury.

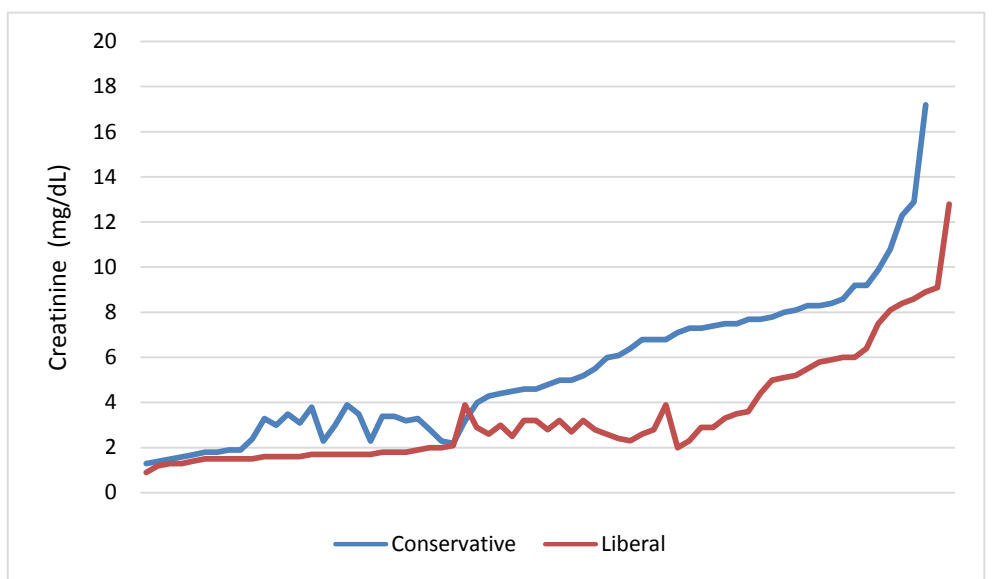

Figure 3. Comparison of Peak Creatinine measurements of all study subjects. 


\subsection{Major Outcomes}

Main outcome variables are shown in Table 2. Results are reported according to fluid management strategy. More patients in the liberal fluid group with 38 patients $(55.07 \%)$ were found to have improvement in renal function, as evidenced by improvement in creatinine levels and overall disposition of patients (discharge), as compared to patients in the conservative fluid group with 11 (16.41\%) patients, regardless of whether patients were admitted in the ICU or on the medical floors $(\mathrm{p}=0.002)$. With regards to the different stages of AKI, there was statistical difference ( $\mathrm{p}=0.02$ ) between the 2 groups, with more patients in the liberal group experiencing improvement in their renal function, in all stages of AKI, as compared to the conservative group. However, in patients who underwent renal replacement therapy, 12 (17.91\%) in the conservative group and 9 (13.04) in the liberal group, there was no difference in the proportion of patients who underwent RRT in both groups $(p=0.43)$. As for the mortality rate, there were more patients in the conservative group $(n=54,80.60 \%)$ who died, noticeably with greater proportion in patients admitted to the ICU $(n=49,98 \%)$, as compared to the liberal group who had 30 patients $(43.48 \%)$ who died, with 24 patients $(70.59 \%)$ admitted in the ICU $(\mathrm{p}<0.001)$. To identify the effect of fluid

Table 2. Main outcome variables.

\begin{tabular}{|c|c|c|c|}
\hline Outcome & $\begin{array}{c}\text { Conservative } \\
\text { fluid management }\end{array}$ & $\begin{array}{l}\text { Liberal fluid } \\
\text { management }\end{array}$ & P Value \\
\hline Improved Renal Function, $\mathrm{n}(\%)$ & $11(16.41)$ & $38(55.07)$ & $<0.001$ \\
\hline ICU & $4(8.00)$ & $12(35.29)$ & 0.002 \\
\hline Non-ICU & $7(41.18)$ & $26(74.29)$ & 0.002 \\
\hline Improved renal function, per stage & & & 0.02 \\
\hline AKI stage 1 & $4(36.36)$ & $24(63.16)$ & \\
\hline AKI stage 2 & $6(54.55)$ & $9(23.68)$ & \\
\hline AKI stage 3 & $1(9.09)$ & $5(13.16)$ & \\
\hline Required RRT, n (\%) & $12(17.91)$ & $9(13.04)$ & 0.43 \\
\hline ICU & $11(22.00)$ & $8(23.53)$ & 0.87 \\
\hline Non-ICU & $1(5.88)$ & $1(2.86)$ & 0.45 \\
\hline Death, n (\%) & $54(80.60)$ & $30(43.48)$ & $<0.001$ \\
\hline ICU & $49(98.00)$ & $24(70.59)$ & $<0.001$ \\
\hline Non-ICU & $5(29.41)$ & $6(17.14)$ & 0.17 \\
\hline Death, per stage & & & 0.28 \\
\hline AKI stage 1 & $7(12.96)$ & $3(10.00)$ & \\
\hline AKI stage 2 & $7(12.96)$ & $8(26.67)$ & \\
\hline AKI stage 3 & $40(74.07)$ & $30(63.33)$ & \\
\hline Improved oxygenation, $\mathrm{n}(\%)$ & $2(3.77)$ & $13(30.95)$ & $<0.001$ \\
\hline
\end{tabular}

ICU, Intensive Care Unit; AKI, Acute Kidney Injury; RRT, Renal Replacement Therapy; n, mean average. 
management on oxygenation, ABGs were analyzed, although this data was limited as not all patients had ABGs done. Between the 2 different strategies of fluid management, improved oxygenation, with 13 (30.95\%) patients characterized by improved respiratory function, facilitating oxygen weaning, was demonstrated in the liberal fluid management group $(\mathrm{p}<0.001)$. These patients were also noted to belong to the group with improved renal function.

\section{Discussion}

Acute kidney injury is well-described in COVID-19 and is associated with high morbidity and mortality, as evidenced by the different retrospective studies done in China, Italy, and the US among others, with an incidence of AKI in hospitalized patients as high as $22 \%$, and $3.2 \%$ requiring renal replacement therapy [16]. Multiple, often co-existing mechanisms have been postulated as a cause of AKI in patients with COVID-19. The most common etiology based on autopsy and biopsy reports is acute tubular injury and lymphocytic infiltration caused by a viral cytopathic effect. In patients requiring mechanical ventilation and vasopressor support, hemodynamic compromise may lead to AKI. Proximal tubular dysfunction has also been demonstrated as well as microangiopathic injury and cytokine-induced podocyte injury. The ARDS-AKI axis, a vicious cycle, where conditions created by dysfunction of one system lead to worsening of the other [17].

With several mechanisms postulated as a cause of AKI, and with a higher percentage of patients developing in-hospital AKI, nondialytic management becomes important in delaying initiation of renal replacement therapy, as these are limited and resources were once stretched thin during the pandemic. In our cohort of 136 patients with COVID-19 respiratory illness and AKI, there was a significant difference in renal outcome, in terms of improvement of renal function in patients receiving liberal fluid management (55.07\%) versus conservative fluid management $(16.41 \%, \mathrm{p} \leq 0.001)$, with more patients in the liberal group having lower peak creatinine before levels improved. This, as well, was associated with improvement in oxygenation, characterized by improvement in respiratory status, facilitating weaning of oxygen supplementation $(\mathrm{p}<0.001)$. As for the different stages of AKI, renal function improved more in patients at lower stages, whether it be from conservative or liberal group $(\mathrm{p}=0.02)$. This data suggests that with more patients in the liberal group having lower creatinine levels and at earlier stages of AKI, that the likelihood of them having a better renal outcome is greater than in the conservative group.

On the other hand, there was no significant difference between the conservative and liberal groups in terms of undergoing renal replacement therapy. Twenty-one of the 136 patients with AKI required RRT and 19 (90\%) of them were admitted to the ICU and mechanically ventilated. In our cohort of stage 3 AKI patients, all 21 (100\%) patients who underwent RRT died, as compared to stage 3 AKI patients who did not undergo RRT, 38 (90.48\%) of the 42 patients 
died $(\mathrm{p}=0.14)$. Thus, there was no statistical difference in mortality rate of patients who underwent renal replacement therapy, regardless of whether they were in the conservative or liberal strategy group. We could thus infer that management in patients with COVID AKI should be initiated early and that safe and judicious nondialytic management should be of utmost importance, to delay or even avoid renal replacement therapy, as this is associated with very high mortality. This includes focusing on one important aspect of preventing AKI, which is fluid management.

The FACTT trial compared a conservative and a liberal fluid management protocol in Acute Respiratory Distress Syndrome (ARDS) patients, with the trial demonstrating benefit to the conservative arm receiving more diuretic medications and less intravenous fluids in lung function, duration of mechanical ventilation and ICU stay. Our findings with COVID patients, however, have demonstrated that keeping patients euvolomic with liberal amounts of fluids, had better outcomes in renal function, oxygenation and mortality. The patients in the liberal arm received fluid boluses and maintenance fluids to prevent worsening of renal function, with conscious effort to avoid volume overload.

Our study has limitations. This study was done in a single-center, community hospital, with more patients in the older population. This limits the general validity of the study. In addition to the population served, resources were limited, which included unavailability of more advanced modalities including Continuous RRT and peritoneal dialysis. Intermittent HD was the only available resource for RRT, and was stretched thin as well during the pandemic. Nevertheless, efforts were done to increase capacity, including additional machines and hiring more staff. Also, as a retrospective study, there was missing information from the collected data. For one, a number of patients were new to the electronic system, with no prior medical records and no baseline Creatinine levels. Also, aside from the fluid therapy, there was no documentation of whether diuretics were given to the patients, which could be an essential component especially in the conservative management group. Urine output was not clearly documented as well, which is part of the KDIGO criteria. Nevertheless, we believe this study is the first of its kind, and thus, opens a lot of possibilities and improvement for future research.

In conclusion, our data report that liberal fluid management in COVID-19 patients with AKI, had better outcomes, in terms of renal function, oxygenation and mortality rate, as compared to patients in the conservative fluid management group. Once patients are started on renal replacement therapy, however, renal and lung outcomes and mortality rate become insignificant between the two groups.

\section{Conflicts of Interest}

The authors declare no conflicts of interest regarding the publication of this paper. 


\section{References}

[1] Cheng, Y., Luo, R., Wang, K., Zhang, M., Wang, Z., Dong, L., et al. (2020) Kidney Disease Is Associated with In-Hospital Death of Patients with COVID-19. Kidney International, 97, 829-838. https://doi.org/10.1016/j.kint.2020.03.005

[2] Zhou, F., Yu, T., Du, R., Fan, G., Liu, Y., Liu, Z., et al. (2020) Clinical Course and Risk Factors for Modality of Adult Inpatients with COVID-19 in Wuhan, China: A Restrospective Cohort Study. The Lancet, 395, 1054-1062.

https://doi.org/10.1016/S0140-6736(20)30566-3

[3] Guan, W.J., Ni, Z.Y., Hu, Y., Liang, W.H., Ou, C.Q., He, J.X., et al. (2020) Clinical Characteristics of Coronavirus Disease 2019 in China. The New England Journal of Medicine, 382, 1708-1720.

[4] Wang, D., Hu, B., Hu, C., Zhu, F., Liu, X., Zhang, J., et al. (2020) Clinical Characteristics of 138 Hospitalized Patients with 2019 Novel Coronavirus-Infected Pneumonia in Wuhan, China. JAMA, 323, 1061-1069. https://doi.org/10.1001/jama.2020.1585

[5] Chen, N., Zhou, M., Dong, X., Qu, J., Gong, F., Han, Y., et al. (2020) Epidemiological and Clinical Charactersitics of 99 Cases of 2019 Novel Coronavirus Pneumonia in Wuhan China: A Descriptive Study. The Lancet, 395, 507-513. https://doi.org/10.1016/S0140-6736(20)30211-7

[6] Pei, G., Zhang, Z., Peng, J., Liu, L., Zhang, C., et al. (2020) Renal Involvement and Early Prognosis in Patients with COVID-19 Pneumonia. Journal of American Society of Nephrology, 31, 1157-1165. https://doi.org/10.1681/ASN.2020030276

[7] Richardson, S., Hirsch, J.S., Narasimhan, M., et al. (2020) Presenting Characteristics, Comorbidities, and Outcomes among 5700 Patients Hospitalized with COVID-19 in the New York City Area. JAMA, 323, 2052-2059.

[8] Mohamed, M., Lukitsch, I., Torres-Ortiz, A.E., et al. (2020) Acute Kidney Injury Associated with Coronavirus Disease 2019 in Urban New Orleans. Kidney360, 1, 614-622. https://doi.org/10.34067/KID.0002652020

[9] Arentz, M., Yim, E., Klaff, L., Lokhandwala, S., Riedo, F.X., Chong, M., et al. (2020) Characteristics and Outcomes of 21 Critically ill Patients with COVID-19 in Washington State. JAMA, 323, 1612-1614. https://doi.org/10.1001/jama.2020.4326

[10] Hirsch, J., Ng, J., Ross, D., Sharma, P., Shah, H., Barnett, R., Hazzan, A., Fishbane, S. and Jhaveri, K. (2020) Acute Kidney Injury in Patients Hospitalized with COVID-19. Kidney International, 98, 209-218.

https://doi.org/10.1016/j.kint.2020.05.006

[11] Sise, M., Baggett, M., Shepard, J., Stevens, J. and Rhee, E. (2020) Case 17-2020: A 68-Year-Old Man with Covid-19 and Acute Kidney Injury. The New England Journal of Medicine, 382, 2147-2156. https://doi.org/10.1056/NEJMcpc2002418

[12] Zahid, U., Ramachandran, P., Spitalewitz, S., et al. (2020) Acute Kidney Injury in COVID-19 Patients: An Innercity Hospital Experience and Policy Implications. American Journal of Nephrology, 51, 786-796. https://doi.org/10.1159/000511160

[13] Nadim, M., Forni, L., Mehta, R., et al. (2020) COVID-19-Associated Acute Kidney Injury: Consensus Reports of the 25th Acute Disease Quality Initiative (ADQI) Workgroup. Nature Reviews, 16, 747-764.

[14] Wiedemann, H., Wheeler, A., Bernard, G., et al. (2006) Comparison of Two Fluid-Management Strategies in Acute Lung Injury. New England Journal of Medicine, 354, 2564-2575.

[15] Joannidis, M., Forni, L., Klein, S., et al. (2020) Lung-Kidney Interactions in Critically Ill Patients: Consensus Report of the Acute Disease Quality Initiative (ADQI) 
21 Workgroup. Intensive Care Medicine, 46, 654-672.

[16] Shaikh, S., Umemoto, G. and Vijayan, A. (2020) Management of Acute kidney injury in Coronovirus Disease 2019. Advances in Chronic Kidney Disease, 27, 377-382. https://doi.org/10.1053/j.ackd.2020.08.002

[17] Kant, S., Menez, S., Hanouneh, M., et al. (2020) The COVID-19 Nephrology Compendium: AKI, CKD, ESKD and Transplantation. BMC Nephrology, 21, Article No. 449. https://doi.org/10.1186/s12882-020-02112-0 